\title{
Kinetics of spontaneous change in the localized motions of $D$-sorbitol glass
}

\author{
G. Power and J. K. Vij ${ }^{\text {a) }}$ \\ Institute of Advanced Materials, Department of Electronic and Electrical Engineering, Trinity College, \\ Dublin 2, Ireland \\ G. P. Joharib) \\ Department of Materials Science and Engineering, McMaster University, Hamilton, \\ Ontario L8S 4L7, Canada
}

(Received 6 October 2005; accepted 5 January 2006; published online 17 February 2006)

\begin{abstract}
The dielectric relaxation spectra of $D$-sorbitol glass have been studied in real time during annealing at $221.1 \mathrm{~K}$, which is $47 \mathrm{~K}$ below its $T_{g}$ of $268 \mathrm{~K}$. As the glass structurally relaxes during annealing, features of the Johari-Goldstein ( $\mathrm{JG})$ relaxation change with time: (i) the relaxation strength decreases, (ii) the relaxation peak at $48 \mathrm{~Hz}$ shifts to a higher frequency, and (iii) the relaxation spectra become narrower. All seem to follow the relation $p \propto \exp \left[-(k t)^{n}\right]$, where $p$ is the magnitude of a property, $k$ the rate constant, and $t$ the time. The parameter $n$ may well be less than 1 , but this could not be ascertained. It is proposed that shift of the relaxation peak to a higher frequency and narrowing of the relaxation spectra occur when local, loosely packed regions of molecules in the glass structure collapse nonuniformly and the relaxation time of some of the molecules in the collapsed state becomes too long to contribute to the JG-relaxation spectra. Consequently, the half width of the spectra decreases, and the relaxation peak shifts to a higher frequency. Molecules whose diffusion becomes too slow after the local regions' collapse would contribute to the $\alpha$-relaxation spectra and thus the net relaxation strength would increase on structural relaxation. It is argued that these findings conflict with the NMR-based conclusions that motion of all molecules in the glass and supercooled liquid contributes to the faster relaxation process. () 2006 American Institute of Physics. [DOI: 10.1063/1.2171195]
\end{abstract}

\section{INTRODUCTION}

Thermally activated fast motions or $\beta$ relaxations in ultraviscous liquids and glasses of molecular substances have attracted much attention in recent years. A topical review of the earlier studies on this subject has been provided by Ngai and Paluch. ${ }^{1}$ These motions have been seen as the terminator of the (molecular) cage motions, ${ }^{2}$ and a precursor of the $\alpha$-relaxation process. ${ }^{3-5}$ Although consequences of a $\beta$ relaxation are seen in a variety of properties, features of its dynamics have been generally studied by dielectric ${ }^{1,6-29}$ and mechanical spectroscopy ${ }^{30}$ and by NMR measurements. ${ }^{31,32}$ Dielectric and mechanical spectroscopies have shown a peak in the isothermal loss spectra or a peak in the plot of loss at a fixed frequency against temperature, and a broad inverted sigmoid-shape decrease in the permittivity and increase in the storage modulus. When only a broad shoulder or an apparent wing has been observed instead of a peak, the $\beta$-relaxation peak has been experimentally resolved by aging the glass, ${ }^{21}$ by using high pressures, ${ }^{25}$ by adding a second component, ${ }^{29}$ and by a detailed analysis of the spectra. ${ }^{19}$ To distinguish it from other $\beta$-relaxation processes, e.g., the mode-coupling theory's $\beta$ relaxation and $\beta$ relaxations in-

\footnotetext{
${ }^{a)}$ Electronic mail: jvij@tcd.ie

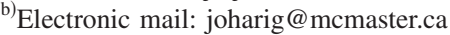

volving intramolecular barriers, the above-mentioned relaxation has been known as the Johari-Goldstein (JG) relaxation. ${ }^{1}$

Molecular origin of the JG relaxation is envisaged in two opposite ways: (i) as localized motions of a fraction of the total number of molecules confined to local regions of low density in the otherwise rigid-disordered structure, $3,6,8,9,22$ and (ii) as faster rotational motion by a small angle prior to the slow motion by a large angle (that shows up as the $\alpha$ relaxation) of all or nearly all molecules in the rigiddisordered structure. ${ }^{31,32}$ In an approach that leaves the mechanism unresolved, Dyre and Olsen ${ }^{24}$ have proposed a minimal model for the JG relaxation. They adapted the Gilroy and Phillips asymmetric double-well potential model, ${ }^{33}$ and allowed the asymmetry of the well depth to become structure dependent. Buchenau and Kahle ${ }^{34}$ and Buchenau ${ }^{35}$ have argued that the same result as obtained by the minimal model may be obtained by a model that attributes the damping to relaxing units distributed around the asymmetry zero with a probability proportional to their weighting factor. The Gilroy-Phillips model has also been reconsidered for the origin of the $\beta$-relaxation process by Coffey et al. ${ }^{36}$ Ngai and co-workers have suggested that the coupling model's $\mathrm{s}^{37-39}$ primitive relaxation ${ }^{38}$ (the exponential relaxation of a single oscillator array, i.e., in the noninteracting case) is related to the JG relaxations in glasses. ${ }^{27,40}$ The primitive relaxation rate may be calculated from the param- 
eters of the $\alpha$-relaxation process, namely, the $\alpha$-relaxation time and the Kohlrausch-Williams-Watts (KWW) stretched exponential parameter, and it has been found to be approximately the same as the JG relaxation rate. ${ }^{27,40}$

Characteristics of the JG relaxation have been recently reviewed. ${ }^{1,2}$ Briefly, (i) the $\alpha$-relaxation process appears to evolve from it at a temperature where its relaxation rate is in the microsecond range, ${ }^{16,41}$ a temperature termed as the Donth temperature (for the merging of the $\alpha$ - and $\beta$-relaxation processes), (ii) the height of the dielectric relaxation peak $\varepsilon^{\prime \prime}{ }_{m \mathrm{JG}}$ and its relaxation strength $\Delta \varepsilon_{\mathrm{JG}}$ decreases on structural relaxation or aging of a glass, ${ }^{8-10,12,24,28}$ (iii) its spectral half width, which is much larger than that of the $\alpha$-relaxation process, increases with a decrease in the temperature, ${ }^{6-10,12-15,23,26,29}$ (iv) its relaxation rate follows an Arrhenius temperature dependence at temperatures $^{6-9,13-16,18,19,25,29-31,42}$ far below the Donth temperature, with an activation energy and preexponent compatible with that for hindered molecular reorientation, (v) the $\Delta \varepsilon_{\mathrm{JG}}$ against temperature plot shows a feature similar to that of enthalpy relaxation on heating, ${ }^{17,18,26}$ and an elbow-shape change in the slope occurs at about $T_{g},{ }^{13,15,23,26}$ the temperature at which onset of the $\alpha$ relaxation begins to contribute and the glass softens to ultraviscous liquid, (vi) the heat capacity contribution begins to increase and a small and broad endothermic feature appears when the JG relaxation becomes kinetically unfrozen on heating some glasses, ${ }^{43}$ (vii) a procedure that decreases $\Delta \varepsilon_{\mathrm{JG}}$ also decreases the linear coefficient of the specific heat at $T<1 \mathrm{~K}$, and increases the thermal conductivity, ${ }^{44}$ and (viii) change in the structure associated with the JG relaxation on aging of silica-based glasses at ambient temperature decreases their specific volume and changes the optical and acoustic properties. ${ }^{45}$

Olsen and co-workers have related the change in JG relaxation features of tripropylene glycol, ${ }^{20}$ pyridine-toluene solution, ${ }^{20}$ polypropylene glycol- $425,{ }^{20}$ D-sorbitol, ${ }^{12}$ and 4,7,10-trioxadecane-1,13-diamine ${ }^{20}$ with the annealing time and temperature to the change in the fictive temperature $T_{f}$, the temperature at which a glass and its equilibrium liquid have the same value of thermodynamic properties, or the temperature at which a glass on heating at an infinitely fast rate would become an equilibrium liquid. ${ }^{46,47}$ As structural relaxation occurs during annealing a glass, its $T_{f}$ decreases. They found that the JG relaxation peak height $\varepsilon^{\prime \prime}{ }_{m, \mathrm{JG}}$ decreased as $T_{f}$ decreased, and the peak frequency $f_{m, \mathrm{JG}}$ increased. Conversely, $\varepsilon^{\prime \prime}{ }_{m, \mathrm{JG}}$ increased as $T_{f}$ increased and $f_{m, \mathrm{JG}}$ decreased. Thus the JG relaxation becomes faster as a glass tends to the equilibrium state. These findings are intriguing because a glass becomes denser as $T_{f}$ decreases, and the relaxation rate is expected to decrease, regardless of whether the JG relaxation involved motions of only a small number of molecules or of all molecules. Although these observations have been described by the "minimal model" within certain approximations and $f_{m, \mathrm{JG}}$ has been found to vary with thermal history of $D$-sorbitol glass (see Fig. 3 and Sec. IV B in Ref. 26), we performed a more detailed study of the JG relaxation spectra isothermally to determine the kinetics of the change in the JG relaxation features with time. We find that the characterizing features of this relaxation change with time according to an equation $p \propto \exp \left[-(k t)^{n}\right]$, where $p$ is a property, $k$ is the rate constant, and $t$ is the time. The parameter $n$ determines the shape of the $p$ against $t$ plot. A review of dielectric relaxation studies of $D$-sorbitol reported until 2002 has appeared in Ref. 26.

\section{EXPERIMENTAL METHODS}

$D$ sorbitol (99.5\% purity) was purchased from Fluka AG and used without further purification. A stainless-steel variable multiplate capacitor with silver-plated electrodes and a nominal maximum air capacitance of $27 \mathrm{pF}$ was used. The temperature was measured by the sensor immersed in the sample. The spectra of the dielectric permittivity $\varepsilon^{\prime}$, and loss $\varepsilon^{\prime \prime}$, were measured in real time over the frequency range of $1 \mathrm{~Hz}-1 \mathrm{MHz}$ by means of a Solartron-Schlumberger SI $1255 \mathrm{~A}$ frequency response analyzer and Chelsea dielectric interface. Further details of the experimental setup are given in Refs. 23 and 26.

$D$-sorbitol was melted at $400 \mathrm{~K}$ in a vacuum oven for 1 day. The capacitor and a Pt $100 \Omega$ resistance temperature sensor were inserted into a $10 \mathrm{~mm}$ diameter, $33 \mathrm{~mm}$ length glass vial containing the molten $D$ sorbitol, ensuring that no air bubbles were trapped. The sample at a temperature of $397 \mathrm{~K}$ was immersed in liquid $\mathrm{N}_{2}$. The insert in Fig. 1(b) shows a plot of temperature against time for a sample after immersion in liquid nitrogen. From this plot, the cooling rate through $T_{g}$ is estimated as $3.0 \mathrm{~K} / \mathrm{s}$. The sample was held in liquid $\mathrm{N}_{2}$ for $8 \mathrm{~min}(\sim 0.5 \mathrm{ks})$ and then transferred to the cryostat where it was heated to $221.1 \mathrm{~K}$, which is $47 \mathrm{~K}$ below the calorimetric $T_{g}$ of $268 \mathrm{~K}$. The sample's temperature was measured at $20 \mathrm{~s}$ intervals. It took $0.5 \mathrm{ks}$ to reach $221.1 \mathrm{~K}$, and $1.3 \mathrm{ks}$ to equilibrate to within $\pm 0.1 \mathrm{~K}$. The sample's dielectric spectra were measured at $221.1 \pm 0.1 \mathrm{~K}$ for a total period of about $16 \mathrm{ks}$, including the $1.3 \mathrm{ks}$ period to reach equilibrium.

\section{RESULTS}

Typical spectra of $\varepsilon^{\prime}$ and $\varepsilon^{\prime \prime}$ measured after periods of 1.5, 5.0, and $16.1 \mathrm{ks}(0.43,1.4$, and $4.5 \mathrm{~h})$ are shown in Figs. 1(a) and 1(b). These show dispersion in $\varepsilon^{\prime}$ and a peak in $\varepsilon^{\prime \prime}$. The glass transition temperature $T_{g}$ of $D$-sorbitol is $268 \mathrm{~K}$ and therefore the sample at $221.1 \mathrm{~K}$ is in the glassy state. The $\varepsilon^{\prime}$ dispersion and $\varepsilon^{\prime \prime}$ peak are due to the JG relaxation, with a small contribution at the low-frequency end that is from the high-frequency tail of the spectra of the kinetically frozen $\alpha$-relaxation process. (Note that the frequency of maximum loss $f_{m}$ of the $\alpha$-relaxation process extrapolated from the Vogel-Fulcher-Tammann equation, $f_{m}=4.92$ $\times 10^{-13} \exp [-884.2 /(221.1-239)],{ }^{18}$ is formally zero for the hypothetical equilibrium liquid at $221.1 \mathrm{~K}$. It is expected to be finite, though extremely small, in a real glassy state of $D$-sorbitol.)

It is evident from the spectra in Fig. 1(a) that the height of the $\varepsilon^{\prime \prime}$ peak spontaneously decreases with time, as has been found in several earlier studies. These spectra were analyzed using the Novocontrol WINFIT program used previously ${ }^{23,26}$ by fitting the real and imaginary parts of the equation for the complex permittivity, 


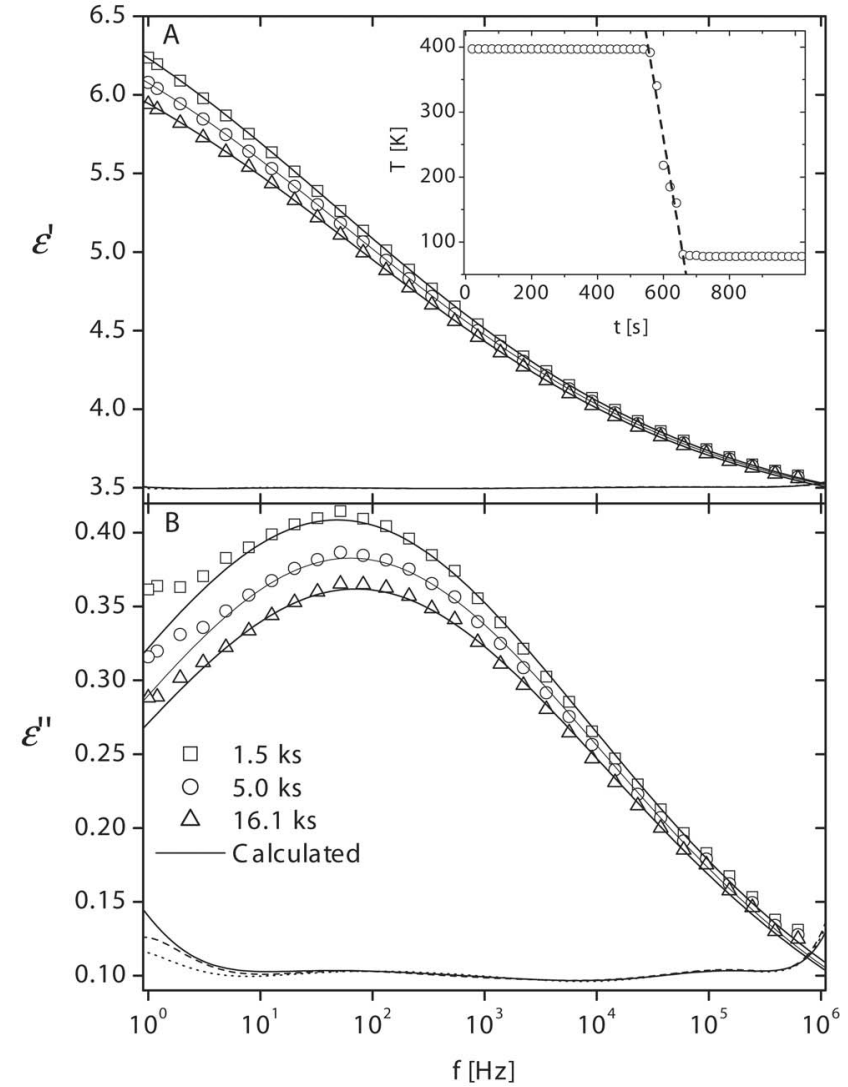

FIG. 1. (A). The measured $\varepsilon^{\prime}$ spectra for the quenched $D$-sorbitol sample kept at $221.1 \mathrm{~K}$ shown at three different times, and (B) the corresponding $\varepsilon^{\prime \prime}$ spectra. The solid lines through the curves are constructed from the $\varepsilon_{\infty}$ and JG-relaxation terms of the fit of Eq. (1) to the data with the values of the parameters as given in the text. Thin solid, dashed, and dotted lines appearing at the bottom of each show the difference between the measured $\varepsilon^{\prime}$ and $\varepsilon^{\prime \prime}$ and the sum of $\varepsilon_{\infty}$ and JG-relaxation terms for $1.5,5.0$, and $16.1 \mathrm{ks}$, respectively. These have been shifted by constants of 3.5 in $\varepsilon^{\prime}$ and 0.1 in $\varepsilon^{\prime \prime}$. These show considerable distortion from a smooth curve, which results mainly from experimental and analytical errors. The insert is the plot of temperature against time during quenching of the $D$-sorbitol sample. The slope of the line near the center of the plot gives $-3.0 \mathrm{~K} \mathrm{~s}^{-1}$ for the cooling rate through $T_{g}$.

$$
\varepsilon^{\prime}-i \varepsilon^{\prime \prime}=\varepsilon_{\infty}+\frac{\Delta \varepsilon_{\alpha}}{\left(1+\left(i \omega \tau_{\alpha}\right)^{\alpha}\right)^{\beta_{\alpha}}}+\frac{\Delta \varepsilon_{\mathrm{JG}}}{1+\left(i \omega \tau_{\mathrm{JG}}\right)^{\alpha_{\mathrm{JG}}}}
$$

where $i=(-1)^{1 / 2}, \omega$ is the angular frequency, $\varepsilon_{\infty}$ is the limiting high-frequency permittivity of the orientation polarization, $\Delta \varepsilon$ is the dielectric strength, and $\tau$ is the relaxation time. The parameters $\alpha$ and $\beta$ define empirically the shape of the spectra which may be related to the distribution of relaxation times. The subscripts $\alpha$ and JG denote the respective relaxation processes, and $\alpha_{\mathrm{JG}}$ is the JG relaxation's symmetric broadness parameter. The high-frequency tail of the $\alpha$-relaxation process extends in the frequency range of our study shown in Fig. 1 , and as $\Delta \varepsilon_{\alpha}$ and $\tau_{\alpha}$ increase when the glass densifies with time, the contribution to $\varepsilon^{\prime \prime}$ in the lowfrequency range of our measurements decreases. This contribution needs to be subtracted from the measured spectra, and in order to do so $\Delta \varepsilon_{\alpha}, \tau_{\alpha}$, and the shape parameters $\alpha_{\alpha}$ and $\beta_{\alpha}$ in the nonequilibrium glassy state at $221.1 \mathrm{~K}$ are needed. Since the $\alpha$-relaxation contribution is no more than $\sim 11 \%$ of the net $\varepsilon^{\prime \prime}$ and less than $0.1 \%$ of the $\varepsilon^{\prime}$ at the lowest fre- quency of $1 \mathrm{~Hz}$, we neglected this contribution and fitted only the JG relaxation term in Eq. (1) to the right side of the relaxation peak of the spectra, i.e., at higher frequencies. First the fit was made to the $\varepsilon^{\prime \prime}$ spectra and then to the $\varepsilon^{\prime}$ spectra, the latter fit to obtain the value of $\varepsilon_{\infty}$. Since there is a large contribution from the JG relaxation and data are not available at high enough frequencies, we are unable to determine the required background dielectric loss, ${ }^{48,49}$ which needs to be subtracted from the measured $\varepsilon^{\prime \prime}$ data.

Nevertheless, we compare this procedure with one in which a contribution from the $\alpha$-relaxation process evaluated from the first term on the right-hand side of Eq. (1) could be subtracted. For this purpose, we use the plots of the relaxation spectra and the distribution parameters in Figs. 3 and 4 of Nozaki et al. ${ }^{14}$ Accordingly, the extrapolated $\Delta \varepsilon_{\alpha}$ at $221.1 \mathrm{~K}$ is $\sim 57, \alpha_{\alpha}$ is $0.86, \beta_{\alpha}$ is 0.38 , and $\tau_{\alpha}$ at $T_{g}$ of $268 \mathrm{~K}$ is about $100 \mathrm{~s}$. According to the Vogel-Fulcher-Tammann equation, the equilibrium liquid's $\tau_{\alpha}$ at $221.1 \mathrm{~K}$ should be infinite because $T_{0}$ of this equation is $239 \mathrm{~K},{ }^{18}$ but in the nonequilibrium glassy state at $221.1 \mathrm{~K}, \tau_{\alpha}$ is expected to be much less than infinity. By subtracting the JG relaxation's $\varepsilon^{\prime \prime}$ peak we obtain a contribution to the $\varepsilon^{\prime \prime}$ from the $\alpha$ relaxation; $\varepsilon^{\prime \prime}{ }_{\alpha}=0.0396$ at $1 \mathrm{~Hz}$ after a period of $1.5 \mathrm{ks}$ in Fig. 1(b). By using these values, we estimate $\tau_{\alpha}=83.4 \mathrm{Ms}$, and by combining it with the above-given $\alpha$-relaxation parameters, we calculate $\varepsilon_{\alpha}^{\prime}-\varepsilon_{\infty}=0.07$ at $1 \mathrm{~Hz}$. The corresponding value in Fig. 1(a) is 0.0035 .

Thus, we determined the parameters $\Delta \varepsilon_{\mathrm{JG}}, \tau_{\mathrm{JG}}$, and $\alpha_{\mathrm{JG}}$. The solid lines through the measured data at each time in Figs. 1(a) and 1(b) were constructed by using the following values of the fit parameters in Eq. (1). (i) At $1.5 \mathrm{ks}, \Delta \varepsilon_{\mathrm{JG}}$ $=4.08, \tau_{\mathrm{JG}}=3.29 \mathrm{~ms}, \quad \alpha_{\mathrm{JG}}=0.252$, and $\varepsilon_{\infty}=3.24$. (ii) At $5.0 \mathrm{ks}, \Delta \varepsilon_{\mathrm{JG}}=3.77, \tau_{\mathrm{JG}}=2.49 \mathrm{~ms}, \alpha_{\mathrm{JG}}=0.255$, and $\varepsilon_{\infty}=3.25$. (iii) At $16.1 \mathrm{ks}, \Delta \varepsilon_{\mathrm{JG}}=3.59, \tau_{\mathrm{JG}}=2.23 \mathrm{~ms}, \alpha_{\mathrm{JG}}=0.253$, and $\varepsilon_{\infty}=3.24$. We converted $\tau_{\mathrm{JG}}$ to the frequency of maximum $\operatorname{loss} f_{m, \mathrm{JG}}=1 / 2 \pi \tau_{\mathrm{JG}}$.

The quantities $\Delta \varepsilon_{\mathrm{JG}}, f_{m, \mathrm{JG}}$, and $\alpha_{\mathrm{JG}}$ for $D$-sorbitol at 221.1 K are plotted against time in Figs. 2(a)-2(c), respectively. It is evident that $\Delta \varepsilon_{\mathrm{JG}}$ in Fig. 2(a) decreases with time from 4.1 to 3.6 after an aging period of $16 \mathrm{ks}$. The equation that best fits the data is

$$
\begin{aligned}
\Delta \varepsilon_{\mathrm{JG}}(t)= & \Delta \varepsilon_{\mathrm{JG}}(t \rightarrow 0)-\left[\Delta \varepsilon_{\mathrm{JG}}(t \rightarrow 0)-\Delta \varepsilon_{\mathrm{JG}}(t \rightarrow \infty)\right] \\
& \times\left[1-\exp -(k t)^{n}\right]
\end{aligned}
$$

with $\Delta \varepsilon_{\mathrm{JG}}(t \rightarrow 0)=4.22, \Delta \varepsilon_{\mathrm{JG}}(t \rightarrow \infty)=3.57$, and the rate constant $k=2.05( \pm 0.08) \times 10^{-4} \mathrm{~s}^{-1}$, when the fitting value of $n$ is 1. The values of $\Delta \varepsilon_{\mathrm{JG}}(t \rightarrow 0)$ and $\Delta \varepsilon_{\mathrm{JG}}(t \rightarrow \infty)$ are 4.73 and 3.48 and $k$ is $3.7( \pm 0.3) \times 10^{-4} \mathrm{~s}^{-1}$ when the fitting value of $n$ is 0.5 . Several curves where Eq. (2) is fitted to the data with different values of $n$ are shown in Fig. 2(a).

In contrast, $f_{m, \mathrm{JG}}$ in Fig. 2(b) increases with time, and the equation that best fits the data in Fig. 2(b) is

$$
\begin{aligned}
f_{m, \mathrm{JG}}(t)= & f_{m, \mathrm{JG}}(t \rightarrow 0)+\left[f_{m, \mathrm{JG}}(t \rightarrow \infty)-f_{m, \mathrm{JG}}(t \rightarrow 0)\right] \\
& \times\left[1-\exp -(k t)^{n}\right],
\end{aligned}
$$

with $f_{m, \mathrm{JG}}(t \rightarrow 0)=43.4 \mathrm{~Hz}, f_{m, \mathrm{JG}}(t \rightarrow \infty)=72.3 \mathrm{~Hz}$, and the rate constant $k=1.96( \pm 0.16) \times 10^{-4} \mathrm{~s}^{-1}$ when the fitting value of $n$ is $1 . f_{m, \mathrm{JG}}(t \rightarrow 0)=21.7 \mathrm{~Hz}, f_{m, \mathrm{JG}}(t \rightarrow 0)=77.0 \mathrm{~Hz}$, and 


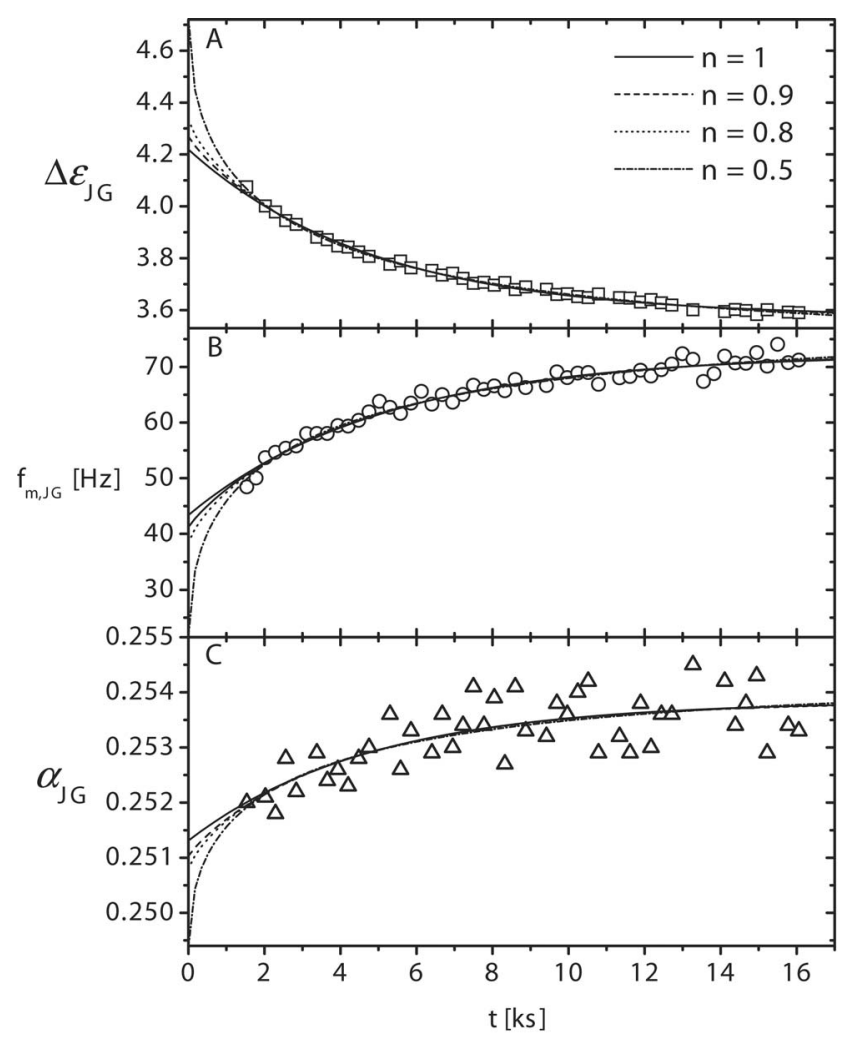

FIG. 2. Plot of (A) $\Delta \varepsilon_{\mathrm{JG}}$, (B) $f_{m, \mathrm{JG}}$, and (C) $\alpha_{\mathrm{JG}}$ against time for the quenched $D$-sorbitol sample kept at $221.1 \mathrm{~K}$. The lines in each panel are fits of Eqs. (2)-(4), respectively to the $\Delta \varepsilon_{\mathrm{JG}}, f_{m, \mathrm{JG}}$, and $\alpha_{\mathrm{JG}}$ vs aging time data for several different values of $n$ as indicated in the figure. For the single exponential case $(n=1)$, the line fitted to the $\Delta \varepsilon_{\mathrm{JG}}$ is given by $\Delta \varepsilon_{\mathrm{JG}}(t)$ $=4.22-(4.22-3.57)\left[1-\exp \left(-2.05 \times 10^{-4} t\right)\right]$. The line fitted to $f_{m, \mathrm{JG}}$, is for the equation $f_{m, \mathrm{JG}}(t)=43.4+(72.3-43.4)\left[1-\exp \left(-1.96 \times 10^{-4} t\right)\right] \quad$ and the line fitted to $\alpha_{\mathrm{JG}}$ is for the equation $\alpha_{\mathrm{JG}}(t)=0.251+(0.254-0.251)$ $\times\left[1-\exp \left(-2.1 \times 10^{-4} t\right)\right]$.

$k=3.3( \pm 0.7) \times 10^{-4} \mathrm{~s}^{-1}$ when the fitting value of $n$ is 0.5 . Fit curves for different $n$ values are also shown. We also note that $f_{m, \mathrm{JG}}$ for $D$-sorbitol after aging for $16 \mathrm{ks}$ is $71.2 \mathrm{~Hz}$. The extrapolated value of $f_{m, \mathrm{JG}}$ at $221.1 \mathrm{~K}$ from earlier measurements ${ }^{26}$ is $78.6 \mathrm{~Hz}$. The difference is relatively small and is also within the experimental and analytical errors.

Finally, the $\alpha_{\mathrm{JG}}$ plot in Fig. 2(c) shows that it also increases with time. The line drawn through the data points in Fig. 2(c) was calculated from the equation,

$$
\begin{aligned}
\alpha_{\mathrm{JG}}(t)= & \alpha_{\mathrm{JG}}(t \rightarrow 0)+\left[\alpha_{\mathrm{JG}}(t \rightarrow \infty)-\alpha_{\mathrm{JG}}(t \rightarrow 0)\right] \\
& \times\left[1-\exp -(k t)^{n}\right],
\end{aligned}
$$

with $\alpha_{\mathrm{JG}}(t \rightarrow 0)=0.251, \alpha_{\mathrm{JG}}(t \rightarrow \infty)=0.254$, and the rate constant $k=2.1( \pm 0.2) \times 10^{-4} \mathrm{~s}^{-1}$ when the fitting value of $n$ is 1 . $\alpha_{\mathrm{JG}}(t \rightarrow 0)=0.249, \quad \alpha_{\mathrm{JG}}(t \rightarrow \infty)=0.254$, and $k=4 \times 10^{-4} \mathrm{~s}^{-1}$ when the fitting value of $n$ is chosen as 0.5 . The uncertainty in $k$ for the fits of the equation to $\alpha_{\mathrm{JG}}$ at low values of $n$ becomes as large as $k$ itself due to the scatter in the data and the narrow range of aging times. As for Figs. 2(a) and 2(b), the fit curves for several different $n$ values are shown.

The $k$ values for the decrease of $\Delta \varepsilon_{\mathrm{JG}}$ and the increase $f_{m, \mathrm{JG}}$ and $\alpha_{\mathrm{JG}}$ obtained when fitting assuming an exponential rate equation are the same within the experimental and analytical errors. In view of the negligible difference between the fits of $\Delta \varepsilon_{\mathrm{JG}}, f_{m, \mathrm{JG}}$, and $\alpha_{\mathrm{JG}}$ obtained for $n=0.5$ and $n=1.0$, the best value of $n$ could not be ascertained. It should, however, be noted that although the kinetics of change in the JG relaxation parameters is a result of irreversible structural relaxation that follows a nonexponential, nonlinear relaxation kinetics, ${ }^{50}$ the apparent kinetics of the change in JGrelaxation parameters would not be necessarily the same as for structural relaxation of an overall thermodynamic or related property observed during annealing of $D$-sorbitol. A further difference between the behavior of $D$-sorbitol and other glasses may stem from the intermolecular hydrogen bonding in the local regions of its glassy state.

It should be noted also that a kinetics with $n=1$ in Eqs. (2)-(4), i.e., an exponential kinetics has indeed been observed for the change in the zero point of the glass thermometer at ambient temperature. This shift of the zero point of a silica-based glass thermometer was reported by Joule in 1884 after his observations made over a period of 38 years, ${ }^{51}$ and it has been verified by other groups since (Ref. 45 may be consulted for details). Joule's data for the shift in zero point have been shown to follow an exponential relation with time with a single rate constant. ${ }^{45}$ It has been interpreted in terms of the localized motions in the silicate network structure and attributed to a decrease in the fictive temperature of the JG relaxation with time. ${ }^{45}$ This interpretation has led to the concept of a second fictive temperature, which is for the JG relaxation and which is subordinated to the usual $T_{f}{ }^{45} \mathrm{It}$ refers to the change in the properties of a glass on aging at a temperature far below $T_{g}$, i.e., for aging at a temperature far below the Vogel-Fulcher-Tammann temperature $T_{0}$. (Note that, in contrast to aging of a glass, structural relaxation occurs relatively close to $T_{g}$ when a glass is annealed, and this occurrence is used for removing internal stresses and gradients of other physical properties in a glass product in an industrial process.) However, the concept of the fictive temperature for the JG relaxation cannot be used here, because at $221.1 \mathrm{~K}$ the JG relaxation is not kinetically frozen. We also note that the temperature dependence of the viscosity of the alkali silicate melt, from which Joule's thermometer had been constructed, deviates much more from the Arrhenius behavior than the viscosity of the $\mathrm{SiO}_{2}$ melt. ${ }^{52}$ Therefore, neither the exponential increase of the zero point of Joule's thermometer glass nor a possible exponential $(n=1)$ decrease in $\Delta \varepsilon_{\mathrm{JG}}$ nor a distribution of the kinetic rate constants $(n<1)$ deduced here can be related to the extent of nonArrhenius temperature dependence of the viscosity of the melt. The reason is that viscosity of a melt is related to the overall structural or $\alpha$-relaxation process and not to the localized kinetics in which a glass does not deform or flow.

\section{DISCUSSION}

Structural relaxation has also been described in terms of the change in the fictive temperature $T_{f}$. As structural relaxation occurs, $T_{f}$ usually decreases and the macroscopic properties of a glass, such as volume, enthalpy, and entropy decrease. These properties are the sum of contributions from (i) the configurational changes associated with the $\alpha$ - and the JG-relaxation processes and (ii) intermolecular vibrations. Although their thermodynamic features have been difficult to 
separate, their relaxational features can be separated and meaningfully interpreted. The magnitude and the variations of $\Delta \varepsilon_{\mathrm{JG}}, f_{m, \mathrm{JG}}$, and $\alpha_{\mathrm{JG}}$ of a glass are unique at a given density, temperature, and pressure of a glass. When structural relaxation increases the density of a glass these properties change. (Note that these properties also change when the density is increased at a fixed temperature by raising the pressure or by decreasing the temperature at a fixed pressure. But the manner in which $\Delta \varepsilon_{\mathrm{JG}}, f_{m, \mathrm{JG}}$, and $\alpha_{\mathrm{JG}}$ change on increase in density as a result of structural relaxation is distinguished from the manner in which they change on deliberately increasing the density, as discussed earlier. ${ }^{22}$ ) Also, when the effects of both the time and temperature were studied by heating a quenched sample at a fixed rate, ${ }^{23,26}$ it was found that changes in $\Delta \varepsilon_{\mathrm{JG}}, f_{m, \mathrm{JG}}$, and $\alpha_{\mathrm{JG}}$ were partly due to structural relaxation at a fixed temperature and partly due to increase in temperature at a fixed structure. Their respective magnitudes varied with the temperature. For clarity, we discuss the effects of decrease in $T_{f}$ on structural relaxation on these properties separately and consider their implications for our current understanding of the glass structure and relaxation.

\section{A. Decrease in $\Delta \varepsilon_{\mathrm{JG}}$ with time}

The net decrease in $\Delta \varepsilon_{\mathrm{JG}}$ owing to simultaneous time and temperature increase during heating at a fixed rate has been found to be analogous to the decrease in the volume, enthalpy, entropy, and $T_{f} \cdot{ }^{26}$ Here, the monotonic decrease in $\Delta \varepsilon_{\mathrm{JG}}$ with time seems analogous to the decrease in the magnitude of these properties with time. ${ }^{47}$ Although, we cannot ascertain the value of $n$ for the kinetics of irreversible change in the characteristics of the JG relaxation with time, it should be noted that thermodynamic properties and $T_{f}$ of a glass do decrease nonexponentially, i.e, with $n<1$ and the characteristic rate constant for this decrease itself decreases with time. This model is known as the nonexponential, nonlinear structural relaxation model and the exponent $\beta$ in it is used in an equivalent expression, ${ }^{50} \exp -\left(t / \tau_{0}\right)^{\beta}$, where $\tau_{0}$ is the inverse of the rate constant for the irreversible decrease in $T_{f}$ with time.

There is also a difference between this kinetics and the kinetics of the overall structural relaxation described in terms of the decrease in $T_{f}$ and which is related to the structure of the ultraviscous liquid and glass. To elaborate, this difference stems from the fact that the dielectric relaxation strength of a material at a given temperature is proportional to its number density of molecules capable of reorienting and the dipolar orientational correlation of the molecular dipoles, as discussed in this context earlier. ${ }^{10}$ As the density increases on structural relaxation at a fixed temperature, the number of dipoles per unit volume would increase and hence $\Delta \varepsilon_{\mathrm{JG}}$ should increase, but here $\Delta \varepsilon_{\mathrm{JG}}$ instead decreases. Thus it seems that as far as the temperature and time are concerned, $\Delta \varepsilon_{\mathrm{JG}}$ changes in the same qualitative manner as a macroscopic thermodynamic property, but as far as molecular aspects of the dielectric process are concerned, $\Delta \varepsilon_{\mathrm{JG}}$ changes in an opposite manner, i.e., it decreases with an increase in density.

\section{B. Increase in the relaxation rate with time}

In the earlier studies, ${ }^{6,8-10,23}$ it had been found that as a glass becomes denser on structural relaxation and its $T_{f}$ decreases, $f_{m, \mathrm{JG}}$ does not change within the detection limits of the single frequency measurements and the dielectric spectra could not be determined in the required short time in those studies. $^{6,8-10}$ It has now been found ${ }^{12,20,24}$ that when a quenched sample structurally relaxes, $f_{m, \mathrm{JG}}$ increases and $\Delta \varepsilon_{\mathrm{JG}}$ decreases. Our present study seems to confirm this finding. An increase in density caused by any external procedure usually decreases the relaxation rate-this is the basis for vitrification of a liquid by increasing the pressure. Therefore, one expects that $f_{m, \mathrm{JG}}$ would decrease with an increase in density of a glass of a homogeneous structure. For that reason, the observed increase in $f_{m, \mathrm{JG}}$ with an increase in density seems puzzling. One may also consider that a possibly undetected increase in $T_{f}$ in our experiments may have increased $f_{m, \mathrm{JG}}$. But if that were the case, $\Delta \varepsilon_{\mathrm{JG}}$ would have also increased because an increase in $T$ and $T_{f}$ increases $\Delta \varepsilon_{\mathrm{JG}}{ }^{9}$ Instead, we find that $\Delta \varepsilon_{\mathrm{JG}}$ continues to decrease with time.

We inquire whether the increase in $f_{m, \mathrm{JG}}$ on structural relaxation with time can be explained within the precept that loosely packed regions exist in the structure of a glass. Before doing so, we first note that $\alpha_{\mathrm{JG}}$ increases with time in Fig. 2(c), i.e., the distribution of relaxation times becomes narrower, and then consider a mechanism for increase in $f_{m, \mathrm{JG}}$ that would be consistent with simultaneous increase in $\alpha_{\mathrm{JG}}$. As a first approximation, we write this distribution as a sum of discrete relaxation times, and represent the measured $\varepsilon^{\prime \prime}$ spectrum as the sum of the relaxation spectra whose $\varepsilon^{\prime \prime}$-peak frequency is distributed over a range corresponding to the relaxation times. For the purpose of illustrating here, we use a rectangular box distribution of relaxation times between the short limit $\tau_{1}$ and the long-time limit of $\tau_{n}$ and for simplicity we assume that the relaxation strength contribution from each spectra is the same. Now when a local region collapses on structural relaxation, those of its molecules or molecular groups whose relaxation time is close to $\tau_{n}$ would kinetically freeze and thus would cease to contribute to $\Delta \varepsilon_{\mathrm{JG}}$. But those molecules whose relaxation time is close to $\tau_{1}$ would remain largely unaffected, and would continue to contribute to the JG relaxation. This means that the limits of the distribution would change more on the long-time or lowfrequency side of the spectra than on the short-time or the high-frequency side. Consequently, the distribution of relaxation times would appear to become narrower, and in this narrow distribution of the spectra the $\varepsilon^{\prime \prime}$ peak would shift to a higher frequency, i.e., $f_{m, \mathrm{JG}}$ would increase.

To illustrate this occurrence, we did a simple calculation whose results are shown in Fig. 3. We chose a rectangular box distribution of relaxation times defined in the frequency domain, and used 10000 Debye-spectral elements (of single relaxation time), and each element with $\Delta \varepsilon=1 \times 10^{-4}$ and $\varepsilon_{\infty}=0$. The lowest $\varepsilon^{\prime \prime}$-peak frequency of the spectral element of the 10000 Debye spectra with a box distribution was $9.69 \mathrm{mHz}$ and each spectral element was $9.69 \mathrm{mHz}$ higher in $\varepsilon^{\prime \prime}$-peak frequency than the preceding one. The 10000 th el- 


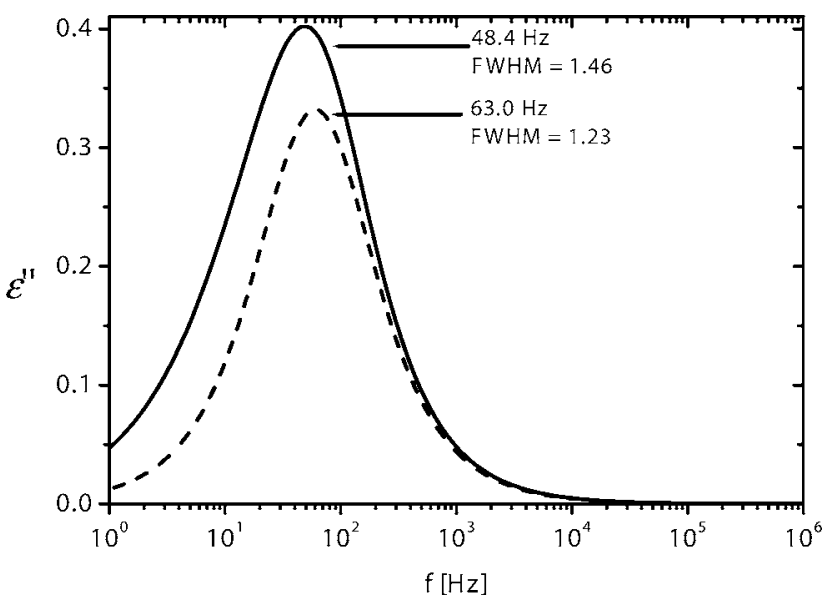

FIG. 3. A simulation of the change in the relaxation spectra with a rectangular box distribution in the frequency domain on kinetic freezing of some of the dipoles. The $\varepsilon^{\prime \prime}$ spectrum was calculated by using a sum of Debyetype relaxations. The spectrum shown by full line was calculated by using $f_{\text {low }}=9.69 \mathrm{mHz}$ and $f_{\text {high }}=96.9 \mathrm{~Hz}$ for the lower and upper bound frequencies of maximum loss of the Debye relaxations in the box distribution and an increment $f_{\text {inc }}$ of $9.69 \mathrm{mHz}$ was chosen, giving a total of 10000 relaxation times. The spectrum shown by the dashed line was calculated by using a lower-frequency limit of $29.1 \mathrm{~Hz}$ and the same increment and upper frequency limit, giving a total of 7001 relaxation times. $\Delta \varepsilon=1 \times 10^{-4}$ and $\varepsilon_{\infty}$ $=0$ in both cases. Note that the removal of relaxing species of the distribution in the range of $9.69 \mathrm{mHz}-29.1 \mathrm{~Hz}$ causes $\varepsilon^{\prime \prime}{ }_{\max }$ and the half width of the spectra to decrease and $f_{m}$ to increase. This is tantamount to removing the dielectric loss contributions from 2999 reorienting species that have become kinetically frozen. The relaxation peak shifts from 48.4 to $63.0 \mathrm{~Hz}$ and the frequency width at half maximum (FWHM) of the spectra decreases from 1.46 to 1.23 decades, showing narrowing of the distribution, as observed here.

ement had a $\varepsilon^{\prime \prime}$-peak frequency of $96.9 \mathrm{~Hz}$. The normalized spectrum calculated for the 10000 elements is shown by the continuous line. It shows a peak at $48.4 \mathrm{~Hz}$. We removed the 2999 Debye-spectral elements from the low-frequency (long time) side of the box distribution and this increased the lower limit of the $\varepsilon^{\prime \prime}$-peak frequency of the elements in the box distribution from $9.69 \mathrm{mHz}$ to $29.1 \mathrm{~Hz}$. The dotted line in the plot shows the resulting spectra. It shows a peak at 63.0 Hz. Evidently, removing of the contributions to the lowfrequency side of the spectra shifts the peak to a higher frequency. Moreover, the frequency width at half maximum (FWHM) of the spectra decreases from 1.46 to 1.23 decades. This is a much larger decrease than that observed in Figs. 1(b) and 2(c), where FWHM decreases from 5.98 decades at $1.5 \mathrm{ks}$ to only 5.95 decades at $16.1 \mathrm{ks}$. Nevertheless, it is qualitatively consistent with the observation that $\alpha_{\mathrm{JG}}$ increases with time, or the JG-relaxation spectra of $D$-sorbitol glass becomes narrower with time. We conclude that the increase in $f_{m, \mathrm{JG}}$ with time is concomitant with the decrease in the distribution of times or narrowing of the spectra and decrease in $\Delta \varepsilon_{\mathrm{JG}}$. (Calculations were done also for a $\log$ normal distribution of a Gaussian type, ${ }^{53}$ which yielded similar results, but for simplicity of illustration here, we have shown only the results of the box distribution.)

Since the earlier studies of the JG relaxation had been performed without regard to the structural relaxation effect, differences in the $f_{m}$ values as a result of this effect may lead to different activation energies. Only in the equilibrium liq- uid state would these values be free from time-dependent effects. To obtain such values accurately, empirical equations for the distribution of relaxation times need to be fitted to the data. Unfortunately, the fitting of the data at temperatures close to the Donth temperature, where the rates of the $\alpha$ and the JG relaxations are closely similar, introduces a serious uncertainty in the estimate of the relaxation times.

\section{Molecular aspects of the change in JG-relaxation features with time}

It is conceivable that the change in the intermolecular hydrogen bonding of $D$-sorbitol in the local regions raises the average relaxation rate and makes the spectra narrower. However, if that were the case, the observation here would seem to be specific to $D$-sorbitol. But since similar effects have been observed also for nonhydrogen-bonded glasses, ${ }^{12,24}$ the behavior observed for $D$-sorbitol would seem to be sufficiently general. Therefore it requires a general mechanism based upon the disordered structure of a glass. The decrease in $\Delta \varepsilon_{\mathrm{JG}}$ observed during structural relaxation has been attributed to the collapse of those local regions where molecules are capable of reorienting. ${ }^{10}$ The finding that the relaxation spectra becomes narrower and the peak frequency becomes higher may be explained by examining the details of this collapse. A nonuniform collapse of such a region would increase the relaxation time of a fraction of its total number of molecules. That fraction would then cease to contribute to the JG relaxation, and become part of the matrix in which diffusion of molecules shows up as a very slow $\alpha$-relaxation process. Were this to occur, it would have the consequence of increasing the relaxation strength of the $\alpha$-relaxation process, $\Delta \varepsilon_{\alpha}$, at the expense of $\Delta \varepsilon_{\mathrm{JG}}$. Hence the total relaxation strength $\left(=\Delta \varepsilon_{\alpha}+\Delta \varepsilon_{\mathrm{JG}}\right)$ would continue to increase with an increase in the density on structural relaxation. This effect has not been observed earlier mainly because during the time taken to measure $\Delta \varepsilon_{\alpha}$, structural relaxation occurs and the state of the sample itself changes.

For instructive purposes we express the above-given findings in terms of change in $T_{f}$. As annealing densifies a glass it should increase $\Delta \varepsilon_{\mathrm{JG}}$ and decrease $f_{m}$ until its $T_{f}$ has approached the actual temperature and the state has approached that of its equilibrium liquid. For a quenched glass, $T_{f}>T$, and for a highly annealed glass rapidly heated to a temperature above the equilibrium temperature, $T_{f}<T$. The structure of a glass solely depends on $T_{f}$ and therefore a change in $T_{f}$ is equivalent to a change in the structure. For modeling the observation that $\Delta \varepsilon_{\mathrm{JG}}$ decreases and $f_{m}$ increases as $T_{f}$ decreases and that $\Delta \varepsilon_{\mathrm{JG}}$ increases and $f_{m}$ decreases when $T_{f}$ increases, Dyre and Olsen ${ }^{24}$ have suggested a double-well potential model with the asymmetry and depth of the wells depending on $T_{f}$. This is seen as a minimal model for the JG relaxation. They have found that the model describes the temperature and structural relaxation dependences of both $f_{m}$ and $\varepsilon^{\prime \prime}{ }_{\text {max }}$ of tripropylene glycol fully at temperatures around $T_{g} \cdot{ }^{24}$ Whether or not the asymmetric potential models can incorporate the change in the distribution of relaxation time of the JG relaxation, as observed here, is an issue that we leave for others to examine in the future. 
At a molecular level, the JG relaxation has been attributed to either the reorientation of molecules or of molecular groups in the relatively loosely packed local regions in the structure of a glass, ${ }^{6,8-10,22}$ or alternatively to small-angle motions of all (Avogadro number of) molecules. ${ }^{31,32}$ Arguments have been given to support both views. However, a number of observations that seem inconsistent with the latter view have been overlooked. For example, if all molecules were to rotate within only two sites to produce different arrangements and there was no redundancy of arrangements in the disordered structure, the configurational entropy arising from the JG relaxation would be equal to $R \ln 2$ $(5.76 \mathrm{~J} / \mathrm{mole} \mathrm{K})$, where $R$ is the gas constant. This value is much higher than the experimentally found values for the JG relaxation. ${ }^{54}$ A further observation is that JG relaxation occurs also in metallic glasses. ${ }^{55-58}$ Since atoms in a metallic glass do not rotate, the relaxation would be due to shortrange translational diffusion of atoms. But a thermally activated translational diffusion of all atoms in a metallic glass would lead to its irrecoverable deformation, which has not been observed. Thus the interpretation of the NMR measurements ${ }^{31,32}$ in terms of small-angle motions of all molecules would not be applicable to all types of glasses. Furthermore, a recent paper by Ichitsubo et al. ${ }^{59}$ has described a microstructural model for a glass consisting of a mixture of weakly regions (islands of mobility) and stronglybonded regions.

\section{CONCLUSIONS}

As structural relaxation of $D$-sorbitol glass occurs isothermally, the strength of the JG relaxation decreases, the relaxation rate increases, and the spectrum becomes narrower. These changes occur with time according to a rate kinetics equation of the type $\exp -(k t)^{n}$, in which $n \leqslant 1$, but the value of $n$ could not be ascertained because data at short times required to determine $n$ could not be reliably obtained as structural relaxation itself occurred during measurement of the spectra. Both the shift of the spectra to a higher frequency and the decrease in the half width can be understood in terms of the collapse of local regions of loosely packed molecules. As some of the regions collapse nonuniformly, those molecules in the collapsed regions that already have long relaxation times within the distribution of JG relaxation times become kinetically frozen and cease to contribute to the JG relaxation. Instead, they contribute to the $\alpha$-relaxation process, which grows at the expense of the JG relaxation. As a consequence, the contribution to the low-frequency side of the JG-relaxation spectra decreases, but the contribution to the high-frequency side does not decrease, or decreases much less. Hence, the dielectric loss peak appears to shift to a higher frequency and its half width decreases.

Finally, we note that the resolution of the JG-relaxation spectra from a set of experimental data and the features of the spectra itself depend upon the equations used to fit the $\alpha$-relaxation spectra. This is to be particularly noted since three empirical equations, the Davidson-Cole, the KWW, and the Havriliak-Negami equations, have all been used to fit the relaxation spectra of various substances and in some cases the spectra of the same substance apparently satisfactorily. While a major part of the $\alpha$-relaxation spectra can be described satisfactorily by each of the three equations, the high-frequency range of the spectra calculated from these equations differs significantly. Subtraction of the calculated spectra from the measured spectra at high frequency would produce different features of the residual, or the JGrelaxation spectra.

\section{ACKNOWLEDGMENTS}

One of the authors (J.K.V.) is grateful to INTEL Ireland Ltd. for funding the studentship of another author (G.P.) (from 2000 to 2004) and for in-part funding of the visit of another author (G.P.J.) to Dublin by the SFI overheads investment plan. Another author (G.P.J.) would like to thank Trinity College for hospitality during his stay for this study.

${ }^{1}$ K. L. Ngai and M. Paluch, J. Chem. Phys. 120, 857 (2004).

${ }^{2}$ K. L. Ngai, AIP Conf. Proc. 708, 515 (2004); in Proceedings of the Third International Symposium on Slow Dynamics in Complex Systems, Sendai, Japan, 3-8 November 2003, edited by M. Tokuyama and I. Oppenheim (unpublished).

${ }^{3}$ J. Y. Cavaille, J. Perez, and G. P. Johari, Phys. Rev. B 39, 2411 (1989).

${ }^{4}$ K. L. Ngai, J. Non-Cryst. Solids 274, 155 (2000); Phys. Rev. E 57, 7346 (1998); J. Non-Cryst. Solids 275, 7 (2000).

${ }^{5}$ B. Bagchi, A. Chandra, and S. A. Rice, J. Chem. Phys. 93, 8991 (1990).

${ }^{6}$ G. P. Johari and M. Goldstein, J. Chem. Phys. 53, 2372 (1970).

${ }^{7}$ G. P. Johari and M. Goldstein, J. Chem. Phys. 55, 4245 (1971).

${ }^{8}$ G. P. Johari, J. Chem. Phys. 58, 1766 (1973).

${ }^{9}$ G. P. Johari, Ann. N.Y. Acad. Sci. 279, 117 (1976).

${ }^{10}$ G. P. Johari, J. Chem. Phys. 77, 4619 (1982).

${ }^{11}$ L. Wu and S. R. Nagel, Phys. Rev. B 46, 11198 (1992).

${ }^{12}$ N. B. Olsen, J. Non-Cryst. Solids 235-237, 399 (1998).

${ }^{13}$ S. Yagihara, M. Yamada, M. Asano, Y. Kanai, N. Shinyashiki, S. Mashimo, and K. L. Ngai, J. Non-Cryst. Solids 235-237, 412 (1998).

${ }^{14}$ R. Nozaki, D. Suzuki, S. Ozawa, and Y. Shiozaki, J. Non-Cryst. Solids 235-237, 393 (1998).

${ }^{15}$ A. Kudlik, S. Benkhof, T. Blochowicz, C. Tschirwitz, and E. Rössler, J. Mol. Struct. 479, 201 (1999).

${ }^{16}$ S. Kahle, E. Hempel, M. Beiner, R. Unger, K. Schröter, and E. Donth, J. Mol. Struct. 479, 149 (1999).

${ }^{17}$ H. Wagner and R. Richert, J. Chem. Phys. 110, 11660 (1999).

${ }^{18}$ H. Wagner and R. Richert, J. Phys. Chem. B 103, 4071 (1999).

${ }^{19}$ T. Fujima, H. Frusawa, K. Ito, and R. Hayakawa, Jpn. J. Appl. Phys., Part 2 39, L744 (2000).

${ }^{20}$ N. B. Olsen, T. Christensen, and J. C. Dyre, Phys. Rev. E 62, 4435 (2000).

${ }^{21}$ U. Schneider, R. Brand, P. Lunkenheimer, and A. Loidl, Phys. Rev. Lett. 84, 5560 (2000).

${ }^{22}$ G. P. Johari, J. Non-Cryst. Solids 307-310, 317 (2002).

${ }^{23}$ G. P. Johari, G. Power, and J. K. Vij, J. Chem. Phys. 117, 1714 (2002).

${ }^{24}$ J. C. Dyre and N. B. Olsen, Phys. Rev. Lett. 91, 155703 (2003).

${ }^{25}$ M. Paluch, C. M. Roland, S. Pawlus, J. Ziolo, and K. L. Ngai, Phys. Rev. Lett. 91, 115701 (2003).

${ }^{26}$ G. Power, G. P. Johari, and J. K. Vij, J. Chem. Phys. 119, 435 (2003).

${ }^{27}$ K. L. Ngai, J. Phys.: Condens. Matter 15, S1107 (2003).

${ }^{28}$ H. Yardimci and R. L. Leheny, Europhys. Lett. 62, 203 (2003).

${ }^{29}$ T. Blochowicz and E. A. Rössler, Phys. Rev. Lett. 92, 225701 (2004).

${ }^{30}$ J. Perez, J. Y. Cavaille, and L. David, J. Mol. Struct. 479, 183 (1999).

${ }^{31}$ A. Döß, M. Paluch, H. Sillescu, and G. Hinze, J. Chem. Phys. 117, 6582 (2002).

${ }^{32}$ M. Vogel and E. Rössler, J. Chem. Phys. 114, 5802 (2001); 115, 10883 (2001).

${ }^{33}$ K. S. Gilroy and W. A. Phillips, Philos. Mag. B 43, 735 (1981).

${ }^{34} \mathrm{U}$. Buchenau and S. Kahle, e-print cond-mat/0310548, and references therein.

${ }^{35}$ U. Buchenau, J. Phys.: Condens. Matter 13, 7827 (2001).

${ }^{36}$ W. T. Coffey, Y. P. Kalmykov, S. V. Titov, and J. K. Vij, Phys. Rev. E 72, 011103 (2005). 
${ }^{37}$ K. L. Ngai, Comments Solid State Phys. 9, 127 (1979).

${ }^{38}$ K. Y. Tsang and K. L. Ngai, Phys. Rev. E 54, R3067 (1996).

${ }^{39}$ K. L. Ngai and K. Y. Tsang, Phys. Rev. E 60, 4511 (1999).

${ }^{40}$ K. L. Ngai, J. Phys.: Condens. Matter 11, A119 (1999).

${ }^{41}$ E. Donth, The Glass Transition: Relaxation Dynamics in Liquids and Disordered Materials (Springer, Berlin, 2001).

${ }^{42}$ S. Pawlus, M. Paluch, M. Sekula, K. L. Ngai, S. J. Rzoska, and J. Ziolo, Phys. Rev. E 68, 021503 (2003).

${ }^{43}$ T. Hikima, M. Hanaya, and M. Oguni, J. Mol. Struct. 479, 245 (1999).

${ }^{44}$ G. P. Johari, Phys. Rev. B 33, 7201 (1986).

${ }^{45}$ S. V. Nemilov and G. P. Johari, Philos. Mag. 83, 3117 (2003); ibid. 84, 845 (2004).

${ }^{46}$ A. Q. Tool, J. Am. Ceram. Soc. 29, 240 (1946).

${ }^{47}$ G. W. Scherer, Relaxations in Glass and Composites (Wiley, New York, 1986).

${ }^{48}$ G. P. Johari, J. Chem. Phys. 122, 144508 (2005); 123, 016102 (2005).

${ }^{49}$ G. P. Johari, Phys. Chem. Chem. Phys. 7, 1091 (2005).

${ }^{50} \mathrm{See}$ the review of the subject in, I. M. Hodge, J. Non-Cryst. Solids $\mathbf{1 6 9}$,
211 (1994).

${ }^{51}$ J. P. Joule, Memoir of the Manchester Literary Philosophical Society, 3rd Ser. 3, 292 (1867); The Scientifc Papers of J. P. Joule (London Physical Society, London, 1884), Vol. 1, p. 558.

${ }^{52}$ V. W. Oldekop, Glastech. Ber. 30, 8 (1957).

${ }^{53}$ A. S. Nowick and B. S. Berry, IBM J. Res. Dev. 5, 312 (1961).

${ }^{54}$ G. P. Johari, J. Chem. Phys. 112, 7518 (2000); 112, 8958 (2000).

${ }^{55}$ A. Eisenberg and S. Reich, in Amorphous Materials, edited by R. Douglas and B. Ellis (Wiley, New York, 1972).

${ }^{56}$ J. M. Pelletier, B. Van de Moortèle, and I. R. Lu, Mater. Sci. Eng., A 336, 190 (2002).

${ }^{57}$ J. M. Pelletier and B. Van de Moortèle, Defect Diffus. Forum 203-205, 265 (2002).

${ }^{58}$ P. Rösner, K. Samwer, and P. Lunkenheimer, Europhys. Lett. 68, 226 (2004).

${ }^{59}$ T. Ichitsubo, E. Matasubara, T. Yamamoto, H. S. Chen, N. Nishiyama, J. Saida, and K. Anazawa, Phys. Rev. Lett. 95, 245501 (2005). 